\title{
New Discretization of Gravitational Field
}

\author{
Branko M. Novakovic
}

\begin{abstract}
Quantum Field Theory (QFT) cannot be applied to a gravitational field, because of non-renormalizability. Therefore, instead of quantization, discretization process of gravitational field is applied. In Relativistic Alpha Field Theory (RAFT) there exists a minimal gravitational radius. Each mass at the minimal gravitational radius has a role as black body. Starting with de Broglie wave equation for a matter, it is transformed into the related RAFT model. The all relations are described as the functions of the dimensionless field parameters $\square$ and $\square$ '. Since the field parameters $\square$ and $\square$ ' can be determined in a total vacuum, electromagnetic field and gravitational field, the generalized wave equations for matter are valid in those field. Here the presented theory is applied only to gravitational field. Each of the wave equations is also described in the related discretization form.
\end{abstract}

Index Terms - Relativistic Alpha Field Theory, discretization of gravitation field, minimal gravitational radius, generalized wave equation .

\section{INTRODUCTION}

Starting with the Einstein's duality hypothesis about the light wave and light particle (photon) observations [1], Louis de Broglie introduced the thesis about matter waves [2,3]. It is usually considered that the influence of gravitation can be neglected in quantum transitions (except in a few experiments trying to detect gravitational waves). Well, this is numerically true for the quantum experiments on the Earth. But, in the case of very strong gravitational field the influence of gravitation to the quantum transitions cannot be neglected. Thus, in the General Theory of Relativity we can include the effect of gravity into the relativistic Klein, Gordon and Fock equation [4]. The new generalizations of the de Broglie wave equation, for a matter is derived in this paper in order to be valid for application in RAF-theory.

The relativistic wave equation for particle physics has been formulated by Paul Dirac [5,6]. As it is the well-known, it was the first theory that fully accounts for relativity in the context of quantum mechanics. Problems in quantum mechanics are also considered in references [7,8-27]. Theoretical support is used from the references [14-20,24,26,27].

As we know, Quantum Field Theory (QFT) [32], cannot be applied to a gravitational field, because this field has non-renormalizability property. Therefore, instead of quantization, discretization process of gravitational field is applied. In order to solve it, here Relativistic Alpha Field theory (RAF-theory) [12,13,16,17,19,23,28-31] is employed. In this theory there exists a minimal gravitational radius. Each mass at the minimal gravitational radius has a role as black body. In that case we can have gravitational radiation of gravitational mass.

Branko Novakovic, FSB - University of Zagreb, Luciceva 5, P.O.B. 509, 10000 Zagreb, Croatia
Here it is started by the generalization of de Broglie wave equation. The mentioned generalization is done by including the dimensionless field parameters $\alpha$ and $\alpha^{\prime}$ into the all relativistic wave equations for matter. The general non-diagonal line element of the RAFT - model [28-30] can be the starting point for derivation of the related wave equations. Finally, the relations that connect gravitational and Planck's parameters, have been presented in [31].

\section{GENERALIZED DE BROGLIE WAVE EQUATION IN RELATIVISTIC ALPHA FIELD THEORY (RAFT)}

It is started with the relations that connect gravitational and Planck's parameters, derived in [31]:

$$
\begin{gathered}
L_{g}=L_{p} \frac{M}{M_{p}}=\sqrt{\hbar G / c^{3}} \frac{M}{M_{p}}, t_{g}=t_{p} \frac{M}{M_{p}}=\sqrt{\hbar G / c^{5}} \frac{M}{M_{p}}, \\
E_{g}=E_{p} \frac{M}{M_{p}}=\sqrt{\frac{\hbar c^{5}}{G}} \frac{M}{M_{p}}, T_{g}=T_{p} \frac{M}{M_{p}}=\sqrt{\frac{\hbar c^{5}}{G k}} \frac{M}{M_{p}} .
\end{gathered}
$$

Here $L_{g}, M, G, t_{g}, E_{g}$, and $T_{g}$ are gravitational length, mass, constant, time, energy and temperature, while $L_{p}, M_{p}, \hbar, t_{p}$, $E_{p}$ and $T_{p}$ are Planck's length, mass, constant, time, energy and temperature, respectively. The amounts of the Planck's parameters are given in the relations:

$$
\begin{gathered}
L_{p}=1.616255 \cdot 10^{-35} \mathrm{~m}, M_{p}=2.176435 \cdot 10^{-8} \mathrm{~kg}, \\
\hbar=6.626070 \cdot 10^{-34} \mathrm{~m}^{2} \mathrm{~kg} \mathrm{~s}^{-1}, t_{p}=5.391245 \cdot 10^{-44} \mathrm{~s}, \\
E_{p}=1,956 \cdot 10^{9} \mathrm{~J}, T_{p}=1.416808 \cdot 10^{32} \mathrm{~K} .
\end{gathered}
$$

The all gravitational parameters can be calculated by multiplying the related Planck's parameters with the ratio of gravitational mass and Planck mass $\left(M / M_{p}\right)(1)$. Appling (1) to the proton in gravitational field, we obtain the following results:

$$
\begin{aligned}
& L_{g p r}=1.242116 \cdot 10^{-54} \mathrm{~m}, M_{p r}=1.672622 \cdot 10^{-27} \mathrm{~kg}, \\
& G=6.67408 \cdot 10^{-11} \mathrm{~m}^{3} \mathrm{~kg}^{-1} \mathrm{~s}^{-2}, t_{g p r}=4.143253 \cdot 10^{-63} \mathrm{~s},(3) \\
& E_{g p r}=1.503215 \cdot 10^{-10} \mathrm{~J}, T_{g p r}=1.088838 \cdot 10^{13} \mathrm{~K} .
\end{aligned}
$$

Compare the gravitational parameters of the proton in (3) with the related Planck's parameters in (2) we can obtain very important conclusions. At the first, gravitational length of the proton is less than Planck length. This confirms that Planck length is not the meaningful minimal length in space-time, because the minimal length belongs to the minimal mass. Further, the gravitational time of the proton is less than Planck time. This is the consequence of the fact that the gravitational length of the proton is less than Planck length.

Generally, following the relations (1), (2) and (3), we can conclude that the all gravitational parameters for the masses less than Planck mass are smaller than the related Planck's parameters. On the contrary, the all gravitational parameters 
for the masses greater than Planck mass are larger than the related Planck's parameters. Also, the ratio of the proton and Planck's parameters are equal to constant:

$$
\frac{M_{p r}}{M_{p}}=\frac{L_{g p r}}{L_{p}}=\frac{t_{g p r}}{t_{p}}=\frac{E_{g p r}}{E_{p}}=\frac{T_{g p r}}{T_{p}}=0.768515 \cdot 10^{-19} .
$$

In order to describe discretization of gravitational field one can start with the principle of minimal gravitational radius, or a minimal gravitational length [31].

Minimal Length Theorem (MLT). The all spherically symmetric bodies (particles) in a gravitational field with mass $M$ should satisfies the following relations:

$$
\begin{gathered}
r_{\text {min }}=\frac{G M}{(1+\kappa) c^{2}}, L_{\text {min }}=2 r_{\text {min }}=\frac{2 G M}{(1+\kappa) c^{2}}, \\
0<\kappa<1, \quad \frac{M}{L_{\text {min }}}=\frac{(1+\kappa) c^{2}}{2 G}=\text { const. } \\
\rho_{\text {rmax }}=\frac{M}{r_{\text {min }}}=\frac{(1+\kappa) c^{2}}{G}=\text { const } .
\end{gathered}
$$

Here $L_{\min }$ and $r_{\min }$ are minimal length and minimal radius of mass $M, c$ is a speed of light in vacuum, $G$ is gravitational constant and $\kappa$ is constant that is between 0 and 1 for closed Universe. Further, $\rho_{r \text { max }}$ is the maximal radial density in a gravitational field at the minimal radius, that is constant and is valid for all gravitational masses in a space time. This is the prediction of RAF theory [28-30].

Gravitational quantum effect for masses less the Plank mass is dominant in the region between $L_{\text {min }}$ and $2 L_{\text {min. }}$. Therefore, discretization of gravitational field shood be determined in the following region:

$$
\begin{gathered}
r_{\text {min }}=\frac{G M}{(1+\kappa) c^{2}}, 0<\kappa<1, L_{\text {min }}=2 r_{\text {min }}=\frac{2 G M}{(1+\kappa) c^{2}}, \\
2 L_{\text {min }}=\frac{4 G M}{(1+\kappa) c^{2}}, 2 L_{\text {min }}-L_{\text {min }}=\frac{2 G M}{(1+\kappa) c^{2}}, \\
\frac{2 L_{\text {min }}-L_{\text {min }}}{L_{d}}=n_{\text {max }}, \quad n=1,2, \ldots, n_{\text {max }} .
\end{gathered}
$$

Parameter $L_{d}$ in (6) denotes distance between discrete points in region $\left(L_{2}-L_{1}\right)$ and $n_{\max }$ is the number of discrete points.

In order to derive a generalized de Broglie wave equation for a matter, one can start with the general invariant relation between frequency and wavelength [3,28]:

$$
\begin{gathered}
\frac{v_{\alpha}^{2}}{c^{2}}-\frac{1}{\lambda_{\alpha}^{2}}=\frac{v_{\alpha}^{\prime 2}}{c^{2}}-\frac{1}{\lambda^{\prime 2}}=\frac{v_{0}^{2}}{c^{2}}, v_{\alpha}=H v_{0}, \frac{1}{\lambda_{\alpha}}=\frac{H v_{0} v_{\alpha}}{c^{2}}, \\
v_{0}=\frac{m_{0} c^{2}}{h}, H=\left(\alpha \alpha^{\prime}-\frac{v^{2}}{c^{2}}+\frac{k\left(\alpha-\alpha^{\prime}\right) c v}{c^{2}}\right)^{-1 / 2} .
\end{gathered}
$$

Here $v_{\alpha}, \lambda_{\alpha}, v_{\alpha}$ and $H$ are frequency, wave length, particle velocity and relativistic parameter in an alpha field theory (RAFT), respectively, while $\alpha$ and $\alpha^{\prime}$ are dimensionless field parameters and $v_{0}$ is the frequency of the particle with rest mass $\mathrm{m}_{0}$ that is standing in an alpha. Further $\hbar$ is Planck's constant, $\mathrm{c}$ is the speed of the light in a vacuum and $\mathrm{k}$ is constant equal (+1) and (-1) [28]. The relations (7) can be discretized as follow:

$$
\begin{gathered}
\frac{v_{\alpha \mathrm{n}}^{2}}{\mathrm{c}^{2}}-\frac{1}{\lambda_{\alpha \mathrm{n}}^{2}}=\frac{v_{\alpha \mathrm{n}}^{\prime 2}}{\mathrm{c}^{2}}-\frac{1}{\lambda_{\alpha \mathrm{n}}^{\prime 2}}=\frac{v_{0}^{2}}{\mathrm{c}^{2}}, \mathrm{n}=1,2 . . \mathrm{n}_{\max }, \\
v_{\alpha \mathrm{n}}=\mathrm{H}_{\mathrm{n}} v_{0}, \frac{1}{\lambda_{\alpha \mathrm{n}}}=\frac{\mathrm{H}_{\mathrm{n}} v_{0} v_{\alpha \mathrm{n}}}{\mathrm{c}^{2}}, v_{0}=\frac{\mathrm{m}_{0} \mathrm{c}^{2}}{\mathrm{~h}}, \\
\mathrm{H}_{\mathrm{n}}=\left(\alpha \alpha_{\mathrm{n}}^{\prime}-\frac{\mathrm{v}_{\mathrm{n}}^{2}}{\mathrm{c}^{2}}+\frac{\mathrm{k}\left(\alpha_{\mathrm{n}}-\alpha_{\mathrm{n}}^{\prime}\right) \mathrm{c} \mathrm{v}_{\mathrm{n}}}{\mathrm{c}^{2}}\right)^{-1 / 2} .
\end{gathered}
$$

Here $\mathrm{n}$ is discrete point and is determined by (6). The parameters $\alpha$ and $\alpha^{\prime}$ in a gravitational field are given by the relations [28-30]:

$$
\begin{gathered}
\alpha_{1}=1-\sqrt{\frac{2 G M}{\mathrm{rc}^{2}}-\left(\frac{G M}{\mathrm{rc}^{2}}\right)^{2}}, \alpha_{1}{ }^{\prime}=1+\sqrt{\frac{2 G M}{\mathrm{rc}^{2}}-\left(\frac{G M}{\mathrm{rc}^{2}}\right)^{2}}, \\
\alpha_{2}=\alpha_{1}{ }^{\prime}, \alpha_{2}{ }^{\prime}=\alpha_{1}, \alpha_{3}=-1-\sqrt{\cdot}, \alpha_{3}{ }^{\prime}=-1+\sqrt{\cdot}, \\
\alpha_{4}=\alpha_{3}{ }^{\prime}, \alpha_{4}{ }^{\prime}=\alpha_{3}, \alpha_{1}-\alpha_{1}{ }^{\prime}=-2 \sqrt{\cdot}, \alpha_{2}-\alpha_{2}{ }^{\prime}=2 \sqrt{\cdot}, \\
\alpha_{3}-\alpha_{3}{ }^{\prime}=-2 \sqrt{\cdot}, \quad \alpha_{4}-\alpha_{4}{ }^{\prime}=2 \sqrt{\cdot} .
\end{gathered}
$$

From the relations (9) we can see that there four solutions of the field parameters $\alpha$ and $\alpha^{\prime}$ in a gravitational field and two solutions of their differences. For a particle motion in $\mathrm{x}$ direction only, particle velocity in an alpha field $\mathrm{v}_{\alpha}$ can be calculated by using the relation [28]:

$$
\begin{gathered}
\mathrm{v}_{\alpha}=\mathrm{v}_{\alpha_{\mathrm{x}}}=\mathrm{v}_{\mathrm{x}}+\mathrm{v}_{\mathrm{f}_{\mathrm{x}}}, \\
\mathrm{v}_{\alpha}=\mathrm{v} \cos \varphi+\mathrm{v}_{\mathrm{f}} \cos \psi=\mathrm{v}_{\mathrm{x}}-\frac{\mathrm{k}\left(\alpha-\alpha^{\prime}\right)_{\mathrm{x}} \mathrm{c}}{2} .
\end{gathered}
$$

If the angels between velocity vectors $\left(\vec{v}, \vec{v}_{\alpha}\right)$ and $\left(\vec{v}_{f}, \vec{v}_{\alpha}\right)$ are equal to zero $\left(\varphi=0\right.$ and $\psi=0$, respectively), then $\mathrm{v}_{\mathrm{x}} \rightarrow \mathrm{v}$ and $\mathrm{k}\left(\alpha-\alpha^{\prime}\right)_{\mathrm{x}} \rightarrow \mathrm{k}\left(\alpha-\alpha^{\prime}\right)$. In that case the relation (10) is transformed into the expression:

$$
\begin{aligned}
& \mathrm{v}_{\alpha}=\mathrm{v}+\mathrm{v}_{\mathrm{f}}=\mathrm{v}-\frac{\mathrm{k}\left(\alpha-\alpha^{\prime}\right) \mathrm{c}}{2}, \\
& \mathrm{v}_{\alpha}=\mathrm{v}+\mathrm{k} \sqrt{\frac{2 G M}{\mathrm{r}}\left(1-\frac{G M}{2 \mathrm{rc}^{2}}\right)} .
\end{aligned}
$$

In the case of a vacuum, without any potential field, the field parameters $\alpha$ and $\alpha^{\prime}$ satisfy the relation $\alpha=\alpha^{\prime}=1$. For that case, the relation (7) is transformed into the well-known invariant relation between frequency and wavelength valid in the Special Relativity:

$$
\begin{gathered}
\frac{v^{2}}{c^{2}}-\frac{1}{\lambda^{2}}=\frac{v^{\prime 2}}{c^{2}}-\frac{1}{\lambda^{\prime 2}}=\frac{v_{0}^{2}}{c^{2}}, v=H_{e} v_{0}, \\
\frac{1}{\lambda}=\frac{H_{e} v_{0} v}{c^{2}}, v_{0}=\frac{m_{0} c^{2}}{h}, H_{e}=\left(1-\frac{v^{2}}{c^{2}}\right)^{-1 / 2} .
\end{gathered}
$$

Further, we shall follow the presentation procedure given in [22-28]. Now, let $\psi_{\alpha}$ be an invariant wave function of a mater in an alpha field. Multiplying the first line in the 
equations (7) by the term $4 \pi^{2} \psi_{\alpha}$ we obtain the following result:

$$
\frac{4 \pi^{2} v_{\alpha}^{2}}{c^{2}} \psi_{\alpha}-\frac{4 \pi^{2}}{\lambda_{\alpha}^{2}} \psi_{\alpha}=\frac{4 \pi^{2} v_{0}^{2}}{c^{2}} \psi_{\alpha}
$$

Here, $\psi_{\alpha}$ is a plane wave propagating in the $\mathrm{x}$ direction only, and described by the well-known function:

$$
\psi_{\alpha}=A \cos 2 \pi\left(v_{\alpha} \mathrm{t}-\frac{\mathrm{x}}{\lambda_{\alpha}}\right) .
$$

Here $\mathrm{A}$ is the wave amplitude. The second partial derivatives of that function on $\mathrm{t}$ and $\mathrm{x}$ are resulted by the following form:

$$
\frac{\partial^{2} \psi_{\alpha}}{\partial \mathrm{t}^{2}}=-4 \pi^{2} v_{\alpha}^{2} \psi_{\alpha}, \frac{\partial^{2} \psi_{\alpha}}{\partial \mathrm{x}^{2}}=-\frac{4 \pi^{2}}{\lambda_{\alpha}^{2}} \psi_{\alpha} .
$$

From the relation (15) one obtains the equations for calculation of $v_{\alpha}^{2}$ and $1 / \lambda_{\alpha}^{2}$ :

$$
v_{\alpha}^{2}=-\frac{1}{4 \pi^{2} \psi_{\alpha}} \frac{\partial^{2} \psi_{\alpha}}{\partial \mathrm{t}^{2}}, \frac{1}{\lambda_{\alpha}^{2}}=-\frac{1}{4 \pi^{2} \psi_{\alpha}} \frac{\partial^{2} \psi_{\alpha}}{\partial \mathrm{x}^{2}} .
$$

After substitution of $v_{\alpha}^{2}$ and $1 / \lambda_{\alpha}^{2}$ from (16) into the equation (7) one obtains the generalized de Broglie wave equation for a matter, valid in an alpha field for $\mathrm{x}$ axis only:

$$
-\frac{1}{c^{2}} \frac{\partial^{2} \psi_{\alpha}}{\partial \mathrm{t}^{2}}+\frac{\partial^{2} \psi_{\alpha}}{\partial \mathrm{x}^{2}}=\frac{4 \pi^{2} v_{0}^{2}}{\mathrm{c}^{2}} \psi_{\alpha}
$$

This relation can be extended to the $\mathrm{x}, \mathrm{y}$ and $\mathrm{z}$-axes, what results with the general invariant wave equation of mater, i.e. the generalized de Broglie wave equation in an alpha field, valid in RAF theory:

$$
\begin{gathered}
-\frac{1}{c^{2}} \frac{\partial^{2} \psi_{\alpha}}{\partial \mathrm{t}^{2}}+\frac{\partial^{2} \psi_{\alpha}}{\partial \mathrm{x}^{2}}+\frac{\partial^{2} \psi_{\alpha}}{\partial \mathrm{y}^{2}}+\frac{\partial^{2} \psi_{\alpha}}{\partial \mathrm{z}^{2}}=\frac{4 \pi^{2} v_{0}^{2}}{\mathrm{c}^{2}} \psi_{\alpha} \\
v_{0}=\frac{\mathrm{m}_{0} \mathrm{c}^{2}}{\mathrm{~h}},
\end{gathered}
$$

Now, one can describe de Broglie wave equation (18) in discrete form:

$$
\begin{gathered}
-\frac{1}{c^{2}} \frac{\partial^{2} \psi_{\alpha_{n}}}{\partial t_{n}^{2}}+\frac{\partial^{2} \psi_{\alpha_{n}}}{\partial x_{n}^{2}}+\frac{\partial^{2} \psi_{\alpha_{n}}}{\partial y_{n}^{2}} \\
+\frac{\partial^{2} \psi_{\alpha_{n}}}{\partial z_{n}^{2}}=\frac{4 \pi^{2} v_{0}^{2}}{c^{2}} \psi_{\alpha_{n}}, n=1,2, . . n_{\max }
\end{gathered}
$$

Here $\mathrm{n}$ is discrete point and is determined by (6). Thus, the relations in (19) described de Broglie wave equation at each discrete point $\mathrm{n}$.

In a vacuum without any potential field, the field parameters $\alpha$ and $\alpha^{\prime}$ satisfy the equation $\alpha=\alpha^{\prime}=1$. Including this condition, the relation (18) is transformed into the well- known de Broglie wave equation of a mater valid in the Special Relativity:

$$
\begin{gathered}
-\frac{1}{c^{2}} \frac{\partial^{2} \psi}{\partial t^{2}}+\frac{\partial^{2} \psi}{\partial x^{2}}+\frac{\partial^{2} \psi}{\partial y^{2}}+\frac{\partial^{2} \psi}{\partial z^{2}}=\frac{4 \pi^{2} v_{0}^{2}}{c^{2}} \psi \\
v_{0}=\frac{m_{0} c^{2}}{h} .
\end{gathered}
$$

The comparison of the relations (18) and (20) confirms that RAFT - model in an alpha field gives the form invariant wave equation related to the wave equation in the Special relativity.

\section{CONCLUSION}

Since Quantum Field Theory (QFT) cannot be applied to a gravitational field, because of non-renormalizability, here discretization procedure of gravitational field is applied Therefore, this paper presented derivation of the generalized de Broglie wave equation in discrete form, valid for gravitational field. Following the Relativistic Alpha Field Theory (RAFT), the all equations are derived as functions of the dimensionless field parameters $\alpha$ and $\alpha^{\prime}$. The initial item for derivation of discrete equations was the general invariant relation between frequency and wavelength. This includes the values of the parameters $\alpha$ and $\alpha^{\prime}$ as functions of gravitational field. Thus, the derived items are valid for a particle in the gravitational field. Finally, instead of the extension of the relativistic alpha field theory for gravitational field to the quantum mechanics, here the generalization of the relativistic wave equations in discrete form is described.

\section{REFERENCES}

[1] G. O. Young, "Synthetic structure of industrial plastics (Book style with paper title and editor)," in Plastics, 2nd ed. vol. 3, J. Peters, Ed. New York: McGraw-Hill, 1964, pp. 15-64.

[2] W.-K. Chen, Linear Networks and Systems (Book style). Belmont, CA: Wadsworth, 1993, pp. 123-135.

[3] H. Poor, An Introduction to Signal Detection and Estimation. New York: Springer-Verlag, 1985, ch. 4

[4] B. Smith, "An approach to graphs of linear forms (Unpublished work style)," unpublished.

[5] E. H. Miller, "A note on reflector arrays (Periodical style-Accepted for publication)," IEEE Trans. Antennas Propagat., to be published.

[6] J. Wang, "Fundamentals of erbium-doped fiber amplifiers arrays (Periodical style-Submitted for publication)," IEEE J. Quantum Electron., submitted for publication.

[7] C. J. Kaufman, Rocky Mountain Research Lab., Boulder, CO, private communication, May 1995.

[8] Y. Yorozu, M. Hirano, K. Oka, and Y. Tagawa, "Electron spectroscopy studies on magneto-optical media and plastic substrate interfaces(Translation Journals style)," IEEE Transl. J. Magn.Jpn., vol. 2, Aug. 1987, pp. 740-741 [Dig. $9^{\text {th }}$ Annu. Conf. Magnetics Japan, 1982, p. 301]

[9] M. Young, The Techincal Writers Handbook. Mill Valley, CA University Science, 1989.

[10] (Basic Book/Monograph Online Sources) J. K. Author. (year, month day). Title (edition) [Type of medium]. Volume(issue). Available: http://www.(URL)

[11] J. Jones. (1991, May 10). Networks (2nd ed.) [Online]. Available: http://www.atm.com

[12] (Journal Online Sources style) K. Author. (year, month). Title. Journal [Type of medium]. Volume(issue), paging if given. Available: http://www.(URL)

[13] R. J. Vidmar. (1992, August). On the use of atmospheric plasmas as electromagnetic reflectors. IEEE Trans. Plasma Sci. [Online]. 21(3). pp. $876-880$. Available: http://www.halcyon.com/pub/journals/21ps03-vidmar 
Acknowledgment

This work was supported by the National Scientific Foundation of Republic of Croatia (grant number: 120-1201842-3048).

Competing interests

The author declares no competing interests.

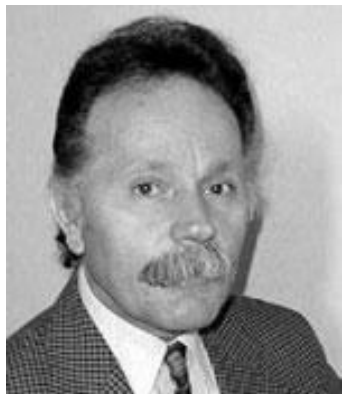

Branko Novakovic is a Professor emeritus at FSB - University of Zagreb,

Croatia. Prof. Novakovic received his $\mathrm{PhD}$ from the University of Zagreb in 1978. His research of interest includes physics, control systems, robotics, neural networks, and fuzzy control. He is author of three books; Relativistic Alpha Field Theory (RAFT, e-book, 2016), Control Methods in Robotics, Flexible Manufacturing Systems and Processes (1990), Control Systems (1985) and the first co-author of a book Artificial Neural Networks (1998). He has published over 240 research papers in his research of interest. 